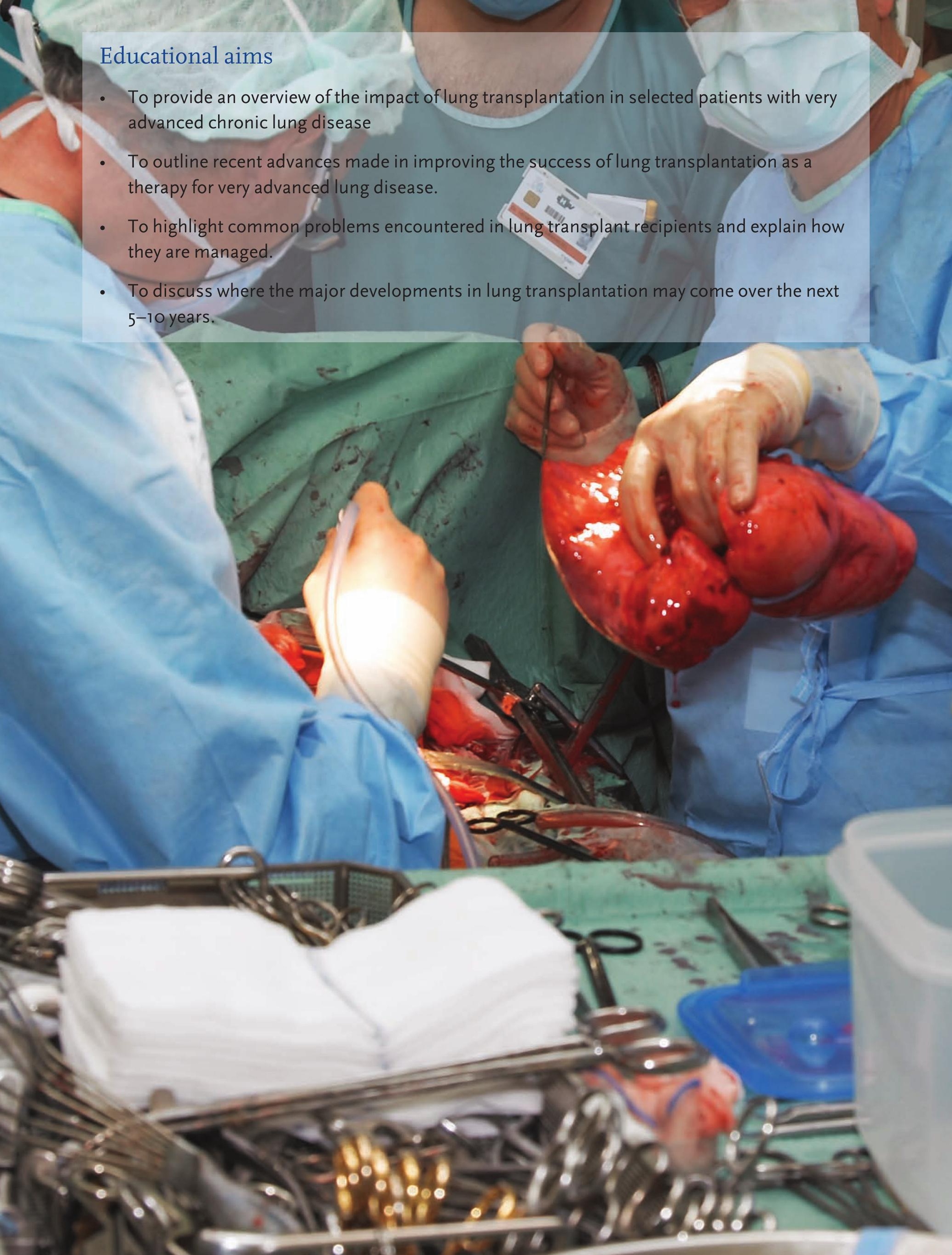




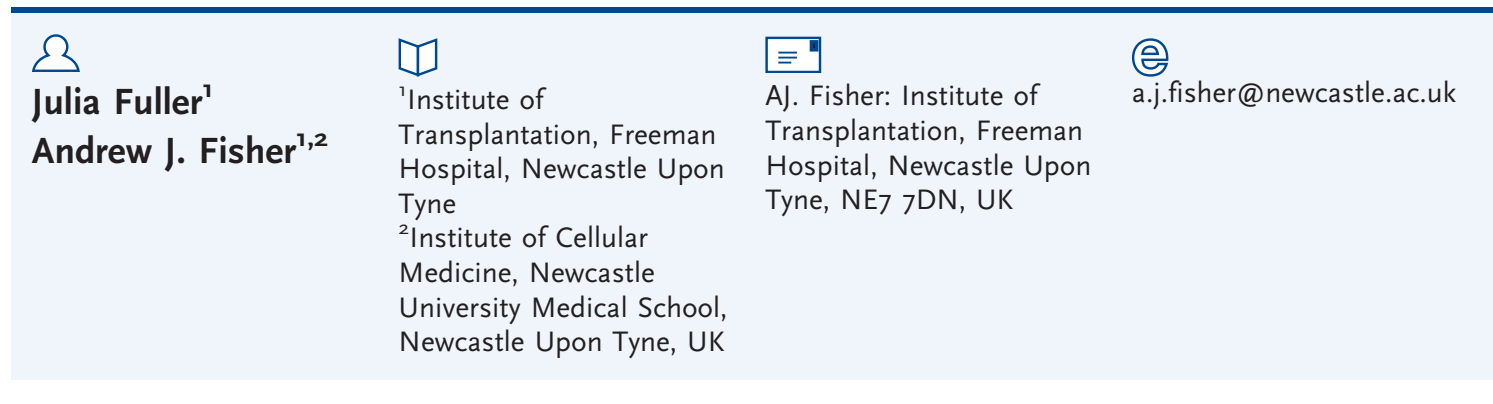

\section{An update on lung transplantation}

\section{Summary}

Over the last 25 years, lung transplantation has developed into a well-established treatment option for selected patients with very advanced lung disease.

In 2010, over a 12-month period, more than 3500 new lung transplant procedures were reported to the International Society of Heart and Lung Transplantation (ISHLT) Registry [1]. In 2011, 1830 lung transplants were performed in the USA [2]. In 2012,690 were performed in the "Eurotransplant" region [3] and a further 170 within the UK [4]. New transplant programmes are now being established around Europe, increasing access to this life saving intervention and benefitting from sharing experience with more established centres. Although worldwide activity has increased year on year over the last decade (fig. 1), demand has also increased meaning the significant shortage of suitable donor lungs accounts for an unacceptably high waiting list mortality rate in many countries.

Clinical experience shared between transplant centres worldwide has helped refine the assessment process so that those added to a waiting list are those who are most likely to benefit. Furthermore, a meticulous approach to posttransplant management in the immediate post-operative period, in the early and long-term has contributed to continually improving long-term survival after lung transplantation.

\section{What does lung transplant offer patients with advanced lung disease?}

Lung transplantation should be viewed as a potential treatment option for highly selected patients with very advanced chronic lung disease who continue to progress despite maximal medical therapy. Identifying those patients with end-stage lung disease who might benefit from lung transplant and referring them promptly for specialist assessment is very important. Referring clinicians play an essential role in identifying patients who might benefit,
Statement of Interest None declared.

HERMES syllabus link: H.1 
but equally need to avoid overwhelming transplant centres with inappropriate referrals which would limit the effectiveness of the centre to assess and list in a timely manner those most likely to benefit.

Lung Transplantation is by no means a cure and should be thought of as another modality of treatment. Life after lung transplantation necessitates use of a large number of medications on a daily basis, frequent hospital visits and the risk of a large list of potential adverse effects due to use of powerful immunosuppression. By finely balancing the protective effects of immunosuppression against lung allograft rejection with the significant toxic effects of these therapies, excellent long-term survival can be achieved. In experienced centres, specific patient groups, such as those with cystic fibrosis (CF), can expect a median survival of well over 10 years with some recipients functioning well more than 20 years after their transplant.

When successful, the procedure is also associated with a marked improvement in quality of life and a proven survival advantage in patients with $\mathrm{CF}$, idiopathic pulmonary fibrosis and pulmonary hypertension. However, in some other indications, including chronic obstructive pulmonary disease (COPD), this remains less clear because a substantial improvement in quality of life may not be matched by an increase in overall survival. Whilst quality of life should be considered in all potential recipients, it is currently unlikely

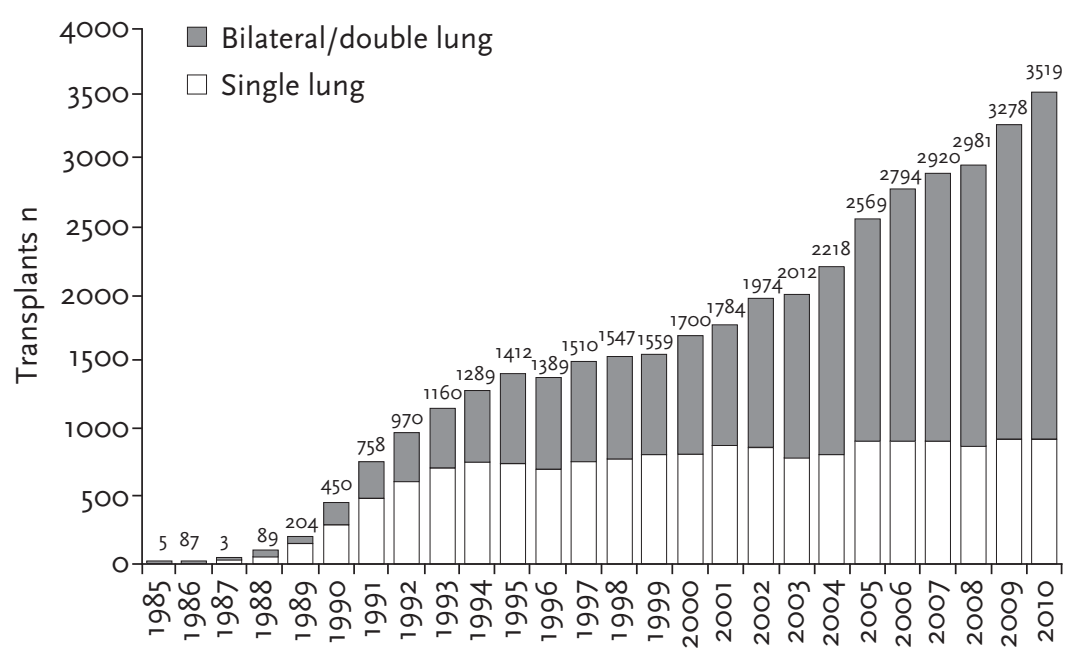

Figure 1

The number of adult lung transplants reported to the ISHLT each year since 1985. Reproduced from [1] with permission from the publisher. that transplantation would be offered solely for this reason due to the shortage of available donor organs.

\section{Who should be referred for lung transplant assessment and when?}

Lung transplantation should generally be considered when a patient's risk of mortality from their lung disease is greater than 50\% within the next 2 years. Prior to this point, the peri-operative mortality risks of transplantation may outweigh the benefits. Identifying this tipping point in risk can be difficult and frequently patients will be reviewed by the transplant centre several times before they are accepted onto the waiting list.

In 2006, the ISHLT produced updated referral and listing guidelines which are still applicable today [5]. Individual centres may apply elements of these guidelines more stringently than others depending on both their experience and their local accessibility to donor organs. It is therefore beneficial to discuss individual cases with your local lung transplant centre in the event of any uncertainty about the suitability of a patient. The guidelines discussed are formed from expert consensus opinion as there is little evidence and no randomised controlled trials to support the recommendations. The general indications for lung transplantation are as follows:

- Advanced lung disease (World Health Organization (WHO) functional class III or IV)

- Lung disease which is progressive and refractory to maximum medical intervention

- Estimated survival chance of $<50 \%$ in 2 years without transplantation

- Patient with capacity to understand the risks and their long-term responsibilities in having a lung transplantation

Disease-specific indications are shown in table 1 but there are also clear contraindications to lung transplantation which are relevant across the range of lung diagnoses (table 2). Some of these may act as absolute contraindications while others are considered more relative. 
What are the disease specific indications for referral for lung transplant assessment?

\section{COPD}

Progressive disease despite maximal medical and potentially surgical intervention with volume reduction surgery in a suitable COPD patient warrants referral for consideration of lung transplantation. The use of the BODE index (body mass index, degree of airway obstruction, dyspnoea, exercise capacity) might be helpful in deciding when to refer [6]. Previous studies have indicated that with a BODE score of 5-6 there is unlikely to be a survival benefit from transplantation at that time. A score of 7-10 indicates a median survival of 3 years and referral to a transplant centre should be considered. As the population from which the BODE score was derived included current smokers and were considerably older than those likely to be eligible for lung transplantation, the relevance of the BODE score in identifying patients suitability remains unproven; but, in the absence of any better prognostic indicator, BODE score can be used to inform a decision to refer. The National Emphysema Treatment Trial which primarily looked at patients suitable for lung volume reduction procedures found a high-risk population with a median survival of 3 years despite optimised medical therapy. These patients had a forced expiratory volume in $1 \mathrm{~S}$ (FEV 1 ) of $<20 \%$ predicted and a transfer capacity of the lung for carbon monoxide (TLCO) of $<20 \%$ predicted with a homogenous distribution of emphysema [7]. Other indicators for referral include hospitalisation with hypercapnic respiratory failure during an acute exacerbation and ongoing cor pulmonale despite adequate long-term oxygen therapy.

Table 1. Disease-specific indication for transplant

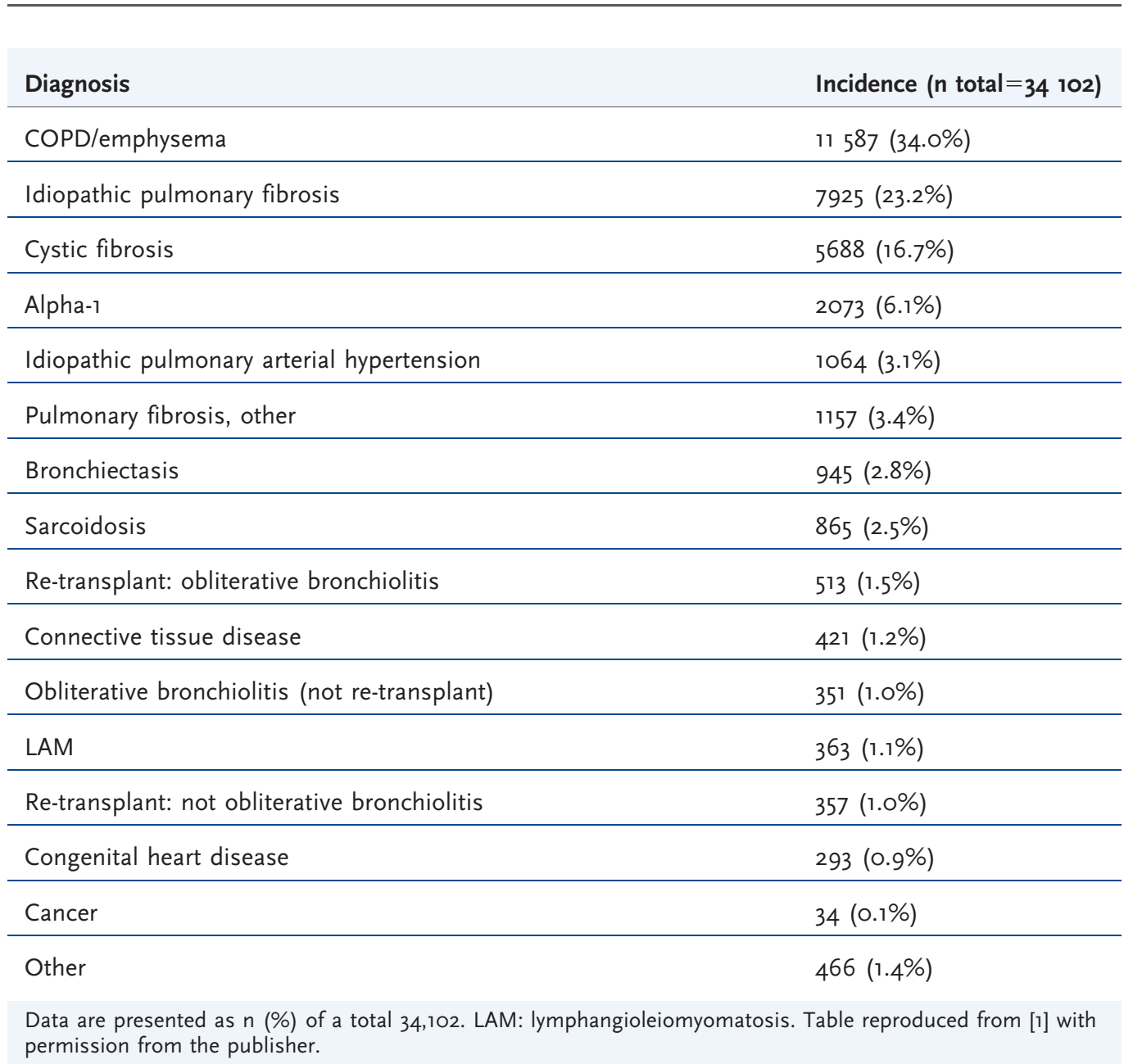


Table 2. Absolute and relative contraindications for transplant

\section{Absolute contraindications to lung transplantation}

Active malignancy within the last 2 to 5 years dependent on tumour type ${ }^{\#}$

Advanced, untreatable disease of any other organ system

Non-curable, chronic extra-pulmonary infection

Significant chest wall or spinal deformity

Documented non-concordance with therapy

Untreatable psychiatric or psychological condition associated

with the inability to concord with treatment

No reliable social support

Substance addiction including smoking (currently or within the

last 6 months)

\section{Relative contraindications to lung transplantation}

Age $>65$ years

Poor functional status with low potential for rehabilitation

Colonisation with highly resistant micro-organisms

Obesity $(\mathrm{BMI}>30)$

Severe symptomatic osteoporosis

Mechanical ventilation

\#: not including basal/squamous cell skin carcinomas.

\section{CF and non-CF bronchiectasis}

There is very limited specific data available for patients with non-CF bronchiectasis and therefore guidance for both conditions is based on experience from patients with CF. Colonisation with organisms such as Pseudomonas aeruginosa, methicillin-resistant Staphylococcus aureus (MRSA), Stenotrophomonas maltophilia and Aspergillus fumigatus are not contraindications to transplant but require carefully managed antimicrobial regimes. In most centres, colonisation with the pan-resistant organisms Mycobacterium abscessus or Burkholderia cenocepacia acts as a contraindication to lung transplantation due to the very high risk of sepsis post-transplant with these organisms [8, 9].

Expert microbiological input to the assessment process is essential as organisms not only infect the lower respiratory tract but also the central airways, nose and sinuses and the risk of re-infection after transplantation from this upper airway reservoir is substantial. The identification of a pre-determined anti-microbial regime effective against organisms present at the time of transplant is very important [5]. It is advised that antimicrobial susceptibility testing is performed regularly whilst waiting for an organ to ensure that the selection given at the time of transplant is effective. Repeated infective exacerbations with resistant organisms, an increasing antibiotic requirement and an increased frequency of hospitalisations are indications for referral when associated with an $\mathrm{FEV}_{1}$ of less than $30 \%$ predicted or with a rapidly declining $\mathrm{FEV}_{1}$. Other indications include an exacerbation that requires intensive care unit admission, oxygen dependency or hypercapnia. Females under the age of 20 with rapid deterioration in their lung function also have a poor prognosis and should be referred early. Patients with CF who have recurrent or refractory pneumothoraces or haemoptysis not controlled by bronchial embolisation should also be referred if otherwise suitable.

\section{Pulmonary fibrosis}

The median survival from initial diagnosis with idiopathic pulmonary fibrosis is only $2.5-3.5$ years. This is reflected in this condition having the highest mortality rate in patients waiting for lung transplantation. Appropriate timing of referral is therefore essential in giving potentially suitable candidates the best chance of being transplanted. Studies have shown the TLCO to be the most reliable marker of deterioration in usual interstitial pneumonia and an absolute value of $<39 \%$ predicted indicates the highest mortality risk. In patients with non-specific interstitial pneumonia this value is set at $<35 \%$ predicted. Other markers associated with poor survival and which should prompt early referral include a drop in vital capacity (VC) of $>10 \%$ in 6 months and oxygen saturations on air $<88 \%$ during a 6 -min walk test. However, studies have suggested that it is the rate of disease progression rather than an absolute cut off in lung function which should guide referral and listing [10]. High-resolution computed tomography (HRCT) images of the lung fields might be useful in guiding the timing of referral as patients with honeycombing as a 
feature of usual interstitial pneumonia have a higher mortality than those without these typical features.

\section{Pulmonary arterial hypertension}

Pulmonary arterial hypertension (PAH) is a progressive disorder characterised by increased pulmonary vascular resistance which, over time, leads to severe right heart failure and ultimately a low cardiac index and death. There have been considerable improvements in medical therapies over the last 10 years for PAH which have altered the rate of disease progression in many patients. As a result fewer PAH patients are being referred for lung transplantation than was historically the case. Those with WHO class III or IV symptoms have the highest mortality risk and studies have also shown a distance of less than $332 \mathrm{~m}$ during a 6-min walk test also indicates a poor prognosis.

Indications for referral include right heart catheterisation showing right atrial pressures of more than $15 \mathrm{mmHg}$ or a cardiac index of less than $2 \mathrm{~L} \cdot \mathrm{min}^{-1} \cdot \mathrm{m}^{-2}$ together with intractable right heart failure despite continuous prostaglandin therapy. In reality, patients now referred are frequently those failing on maximal medical treatment with a highest tolerated dose of intravenous prostaglandin therapy in association with one or two other targeted therapies including phosphodiesterase-5 inhibitors and endothelin receptor antagonists.

\section{Sarcoidosis}

Although not as common an indication for lung transplant as idiopathic pulmonary fibrosis, pulmonary sarcoidosis carries a similar mortality in patients on the waiting list. It is important to remember the potential for considerable extra-pulmonary involvement and indeed bronchiectasis. Triggers for referral should include NYHA class III/IV symptoms, hypoxia at rest and significant secondary pulmonary hypertension.

\section{How are suitable donor lungs identified for patients on the waiting list?}

Matching donor lungs to patients on the waiting list is done traditionally on the basis of size and $A B O$ blood group. The potential donor must have an estimated total lung capacity within certain limits of the measured total lung capacity of the potential recipient. In addition the $A B O$ group of the donor should be compatible with that of the potential recipient. This matching process can be made more difficult if the recipient has developed circulating antibodies against antigens of the human leukocyte antigen (HLA) system. This can happen due to previous blood transfusions or pregnancy but in some cases is of unknown cause. If high concentrations of specific HLA antibodies are present it may mean any potential donor who carries the same HLA on their cells has to be excluded from use. If a potential recipient has numerous preformed anti-HLA antibodies it can lead to a substantial proportion of donors being unsuitable for them due to the risk of hyperacute and subsequent antibody mediated rejection.

When a potential donor lung is matched to a potential recipient, the key question is then whether the donor lung is suitable to be used for transplant. This decision is made using selection criteria which have been agreed by expert consensus [11].

The main limiting factor preventing an increase in the number of lung transplantations being performed is a lack of suitable donors. Very few donor lungs satisfy the criteria of an ideal donor organ (table 3) and if these criteria where strictly adhered to then very few lung transplants would be possible. As a result, lung transplant centres frequently push the boundaries by accepting donor organs which extend the ideal criteria to try to increase activity. Such "extended criteria" lungs as those from donors who have smoked can come with a slight increased risk but this is still less than the risk of mortality on the waiting list by not using such organs [12]. Currently less than $30 \%$ of multi-organ donors provide lungs suitable for transplantation. The remainder are deemed unsuitable due to concerns about their function or risks they may pose to the recipient and therefore are not used.

\section{What can be done to increase the availability of donor lungs for transplantation?}

The mortality rate of those on an active waiting list ranges from 10-30\% depending 
Table 3. ISHLT Consensus for the "ideal" lung donor

\begin{tabular}{l} 
Age $<55$ years \\
ABO compatibility \\
\hline Clear chest radiograph \\
\hline $\mathrm{PaO}_{2}>40 \mathrm{kPa}\left(\mathrm{FiO}_{2} 100 \%\right.$ and $\left.5 \mathrm{CmH}_{2} \mathrm{O} \mathrm{PEEP}\right)$ \\
\hline Smoking history $<20$ pack years \\
Absence of chest trauma
\end{tabular}

No evidence of aspiration or sepsis

No prior cardiopulmonary surgery

Absence of organisms on sputum Gram stain

Absence of purulent secretions at bronchoscopy

on the transplant centre and the country. This could be substantially reduced by increasing the number of suitable donor lungs available. Strategies to achieve this include via legislation in adopting a presumed consent system as is already used in several European countries, extending the criteria for donor suitability even further, resizing donor lungs to fit recipients that would not have been a size match by standard criteria or by performing lobar lung transplantation using lobes donated by living family members or friends. All these approaches have been used successfully in individual centres. There are however two recent developments which offer hope that availability of suitable donor lungs could improve significantly. These are the increasing availability of organs from donors after circulatory death and the use of ex vivo lung perfusion as a means to assess and recondition unsuitable donor lungs into organs suitable for transplantation.

The majority of donor lungs come from donors who have suffered a catastrophic cerebral event and have been declared brainstem dead. The events which happen around the time of brainstem death can have a direct effect on the function of the donor lungs and render a significant proportion unsuitable. However, it is now clear that patients in an ICU who have life supportive treatment withdrawn in a controlled way due to futility and die due to cessation of circulatory support can provide lungs for transplantation. Good outcomes in recipients of lungs from donation after circulatory death (DCD) suggest that this will become an important source of donor organs for the future.

\section{EX vivo perfusion of donor lungs}

SteEn et al. [13] in Sweden were the first to use ex vivo lung perfusion (EVLP) to assess lungs prior to transplant. Initial animal studies led to the first successful report of a human lung transplant where the donor organs had been assessed using ex vivo perfusion prior to the procedure. The technique was then extended to include reconditioning of organs which were initially felt to be unsuitable by improving oxygenation and reducing pulmonary vascular resistance [14]. In 2007, the first successful transplant took place using ex vivo perfusion to improve an initially unusable organ [15].

EVLP involves controlled perfusion and ventilation of the donor lungs over a number of hours. Perfusion is performed using a semiautomated circuit using a protein-rich solution with a high oncotic pressure which helps to remove interstitial lung water and decrease pulmonary oedema. It also limits microthrombi formation and cell aggregation. Ventilation strategies use low tidal volumes and low inspired oxygen concentrations to reduce mechanical stretch and oxidative injury along with sustained positive end expiratory pressures to manage atelectatic areas. Therapeutic bronchoscopy with bronchial lavage in specific areas reduces ventilation-perfusion mismatch avoiding parenchymal damage and also provides samples for microbiological analysis to further direct antimicrobial therapy. Current clinical trials are underway to further assess the use of ex vivo lung perfusion to try to increase the number of organs available for transplant.

\section{How do airway complications present after lung transplantation?}

The anastomosis between the donor and recipient bronchi receives its blood supply from the pulmonary circulation following transplantation and relies on the formation of collateral vessels. In the first few weeks the most serious airway complication relates to dehiscence of the primary anastomosis. This may present with a pneumothorax or 
pneumomediastinum and generally results in severe sepsis. In severe cases, treatment is with re-exploration and reconstruction of the anastomosis (fig. 2). Several weeks after transplant, bronchial strictures can occur at the site of the anastomosis and will tend to present with a decrease in $\mathrm{FEV}_{1}$, dyspnoea and stridor. Bronchoscopic intervention for these includes repeated balloon dilatation but sometimes requires stenting of the airway to relieve symptoms.

\section{What are the common infections seen after lung transplantation?}

Infection is one of the leading causes of morbidity and mortality in the post-transplant period. The responsible organisms can be bacterial, viral or fungal. Table 4 shows some of the infections that are common following lung transplantation and their impact. Cytomegalovirus (CMV) infection is one of the most important in the first few months following lung transplantation. Primary infection can occur in seronegative recipients who receive an organ from a seropositive donor. Recipients who are seropositive can also experience reactivation of latent CMV infection due to the effects of immunosuppression or re-infection with another strain from a seropositive donor. Symptoms may be very non-specific, including fevers and lethargy but may develop into pneumonitis or colitis and can result in multi-organ failure and death. Another important opportunistic infection is Pneumocystis jirovecii which because of the severity of pneumonic infection, necessitates lifelong prophylaxis in many centres.

\section{How is immunosuppressive therapy used after lung transplant?}

The use of powerful immunosuppressive treatment is essential to successful management of the lung transplant recipient. Its use requires a balance between the protective effects against allograft rejection and the adverse effects caused by the toxicity of these therapies. Treatment is started at the time of transplantation and continues life-long thereafter. Ideally, maintenance immunosuppression
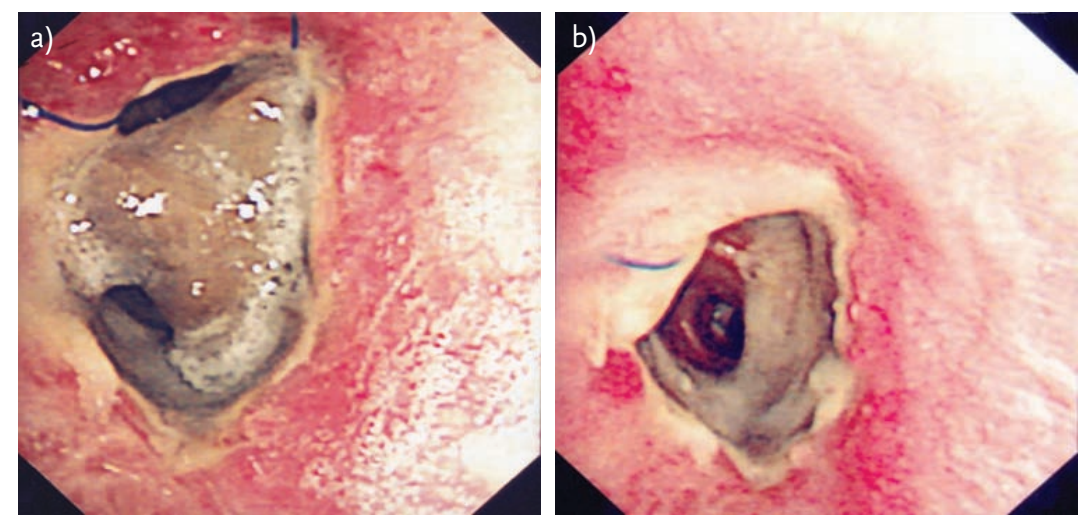

Figure 2

Photographs taken at bronchoscopy to show a) anastomotic narrowing due to organised mucous plug and b) the final result after bronchial toileting.

consists of three drug classes: corticosteroids, calcineurin inhibitors (ciclosporin or tacrolimus) and a cell-cycle inhibitor (mycophenolate mofetil or azathioprine). In addition antibody treatments such as anti-thymocyte globin, basulizumab or alemtuzumab targeting specific leukocyte populations may be used to provide very rapid and in some cases sustained inhibition of lymphocyte activity.

Use of calcineurin inhibitors require regular drug level monitoring in an attempt to ensure plasma levels are therapeutic but also to minimise side effects and toxicity. Calcineurin inhibitors achieve their effect by blocking the protein calcineurin and therefore decreasing the production of interleukin-2 and the subsequent proliferation of T-lymphocytes. They are metabolised via the $\mathrm{p} 450$ system within the liver and therefore their serum level has the potential to be affected by interaction with a wide range of co-administered medications. It is very important for the patient and any treating clinician to be aware that co-prescription of many commonly used medications can lead to an increased risk of toxicity or decreased efficacy of the calcineurin inhibitors.

An example is the use of the macrolide antibiotics claithromycin or erythromycin for the treatment of lower respiratory tract infections or community acquired pneumonia. These can lead to significant calcineurin inhibitor toxicity if co-prescribed; precipitating renal injury and therefore should not be co-prescribed. It is recommended that before any new therapy is given to a lung transplant recipient that potential interactions with their immunosuppression are carefully considered and acted upon. 
Table 4. Common post-transplant infections, their sequelae and management

Post-transplant infection

Clinical features

Pseudomonas aeruginosa
- Can cause asymptomatic colonisation, infective exacerbations or pneumonia

- Occurs anytime but especially in the first year

- Colonised patients often treated with maintenance nebulised antibiotics

- Associated with increased risk of developing Bronchiolitis Obliterans Syndrome

Cytomegalovirus

- Can cause primary infection or re-activation in a previously infected host

- Typically occurs in first few months, though the first year carries greatest risk

- Higher risk recipients given anti-CMV prophylaxis for 3-6 months after transplant

- Associated with increased risk of developing bronchiolitis obliterans syndrome

Epstein-Barr virus

- Re-activation most common within the first year when immunosuppression at highest levels

- Reactivation known to drive development of post-transplant lymphoproliferative disorder (PTLD)

Aspergillus

- Can cause asymptomatic colonisation, airway infection, invasive or cavitatory disease

- Occurs anytime but especially in the first year

- Those colonised with Aspergillus pre-transplant require anti-fungal prophylaxis for 612 weeks after transplant

Data taken from $[16,17]$.

\section{What are the long-term consequences of immunosuppressive therapy use?}

There are a number of adverse effects that are associated with prolonged immunosuppression use especially with calcineurin inhibitors. These include hypertension, which affects over $50 \%$ of lung transplant recipients by the end of the first year and frequently requires pharmacological management [4]. Diabetes mellitus is also common due to both corticosteroid use and the calcineurin inhibitors particularly tacrolimus. The development of renal impairment due to the use of calcineurin inhibitors is a frequent adverse event and can be exacerbated by the hypertension and diabetes that many patients also develop. In some recipients renal impairment can progress into overt renal failure requiring renal replacement therapy and subsequent renal transplantation.

Long-term immunosuppression also significantly increases the risk of malignancy, particularly of skin cancers but also of other solid organ cancers. In the first year, approximately 
$3.5 \%$ of lung transplant recipients will develop some form of malignancy, rising to over $27 \%$ after 10 years [4]. All patients are advised to avoid sun exposure by using sun-block and covering exposed areas. In the post-transplant population, squamous cell skin cancers are much more common than basal cell, contrary to that seen in the general population. Careful monitoring for skin lesions is advised and a low threshold for sampling suspicious lesions should be present.

Post-transplant lymphoproliferative disease (PTLD) is a well-recognised complication of immunosuppression and is seen in about $5 \%$ of lung transplant recipients, which is higher than that seen in other solid organ transplant recipients. It occurs as a result of uncontrolled lymphoid cell proliferation and is frequently but not always driven by EpsteinBarr virus infection [18]. PTLD is most likely to occur in the first year following transplant when immunosuppression is at its highest level and can present either within the transplanted lungs or in other organs, commonly the liver, lymph nodes or the gastrointestinal tract. Symptoms may be non-specific, including fever, weight loss, breathlessness and lethargy. The most common radiological findings are solitary or multiple pulmonary nodules. Treatment regimes include reducing maintenance immunosuppression and use of Rituximab to target CD20 lymphocyte clones. In some cases, when there is no response to the measures above, lymphoma regime chemotherapy is used but patients in this group have a poor prognosis.

\section{How does lung transplant rejection present and what can be done for it?}

Rejection of the transplanted lungs by the recipients' immune system manifests in two main patterns. Acute rejection involves a rapid cell or antibody-driven immune attack on the lung allograft that frequently responds to temporary increases in the potency of immunosuppression used termed "augmentation". Whereas chronic rejection also known as chronic lung allograft dysfunction is a more complex and indolent process that causes progressive loss of graft function with scarring of the lung and increased predisposition to infections.

\section{Acute rejection}

Episodes of acute rejection are common, affecting up to $60 \%$ of lung transplant recipients in their first year [4]. They occur most often within the first three months and are rare after the first year. Symptoms of acute rejection can include breathlessness, cough, hypoxia and more non-specific symptoms, such as fever or lethargy. A chest radiograph may be normal or show new infiltrates or small pleural effusions (fig. 3). Spirometry at this time typically shows a drop in $\mathrm{FEV}_{1}$ of $>10 \%$. If a recipient presents with such symptoms they will require assessment by transbronchial lung biopsy to look for histological evidence of acute rejection.

Histological results are presented as part of an "ABC" scoring system developed by the ISHLT [19], with a number following each letter indicating the presence and severity of abnormalities. A is representative of acute cellular rejection, B of airway inflammation and $\mathrm{C}$ of chronic rejection (table 5).

The mainstay of treatment for acute rejection is significant augmentation of the patient's corticosteroid dose. Depending on the results, high-dose corticosteroids are given intravenously if the acute rejection episode occurs in the first 3 months or orally if the episode occurs thereafter. The dose is then gradually reduced back to a maintenance level over a number of weeks.

\section{Chronic rejection}

Chronic rejection also termed chronic lung allograft dysfunction carries a poorer prognosis

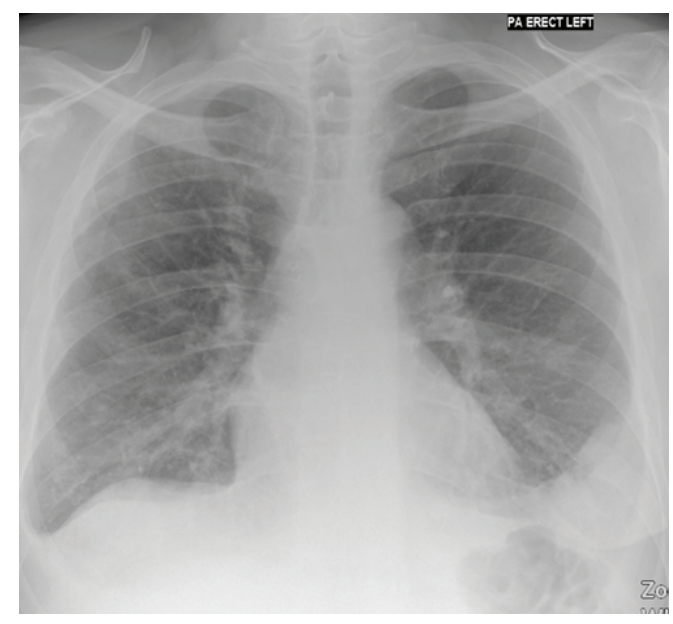

Figure 3

A chest radiograph depicting an episode of acute rejection. 


\section{Educational questions}

1. Contraindications to lung transplantation include:

a. Squamous cell skin carcinoma within the last 2 years

b. Body mass index of 38

c. Single vessel coronary artery disease

d. Aspergillus colonisation of the lungs

e. Current smoker

2. Symptoms or signs of acute rejection may include:
a. Breathlessness
b. Hypoxia
c. Vomiting
d. Pleural effusions
e. Cough

3. Risk factors for the development of chronic rejection include:

a. Gastro-oesophageal reflux disease

b. Recurrent episodes of acute rejection

c. Donor-recipient sex mismatch

d. Good concordance with treatment

e. Infection with cytomegalovirus

4. With regards to lung transplant in general:

a. The number of lung transplants performed worldwide is going down

b. Lung transplant for COPD offers a clear survival benefit

c. Acute rejection frequently responds to high dose corticosteroids

d. Chronic rejection can only be diagnosed from a lung biopsy

e. Lung transplant for Pulmonary Fibrosis offers a clear survival benefit than acute rejection and affects $50-60 \%$ of recipients within 5 years of transplant [20]. Risk factors include recurrent episodes of acute rejection, the presence of HLA-antibodies pretransplant, gastro-oesophageal reflux disease, poor concordance with immunosuppression and bacterial, viral and fungal infections. The most common form of chronic rejection following lung transplant is bronchiolitis obliterans syndrome (BOS). BOS is a clinical diagnosis made after exclusion of other causes for a loss of lung function and the development of progressive airflow obstruction. Obliterative bronchiolitis is the pathological correlate to BOS and is a histological diagnosis characterised by lymphocytic inflammation and disruption to airway epithelium associated with the deposition of fibroblastic tissue that results in partial or complete obliteration of small and medium sized airways [21].

The ISHLT have produced criteria for the diagnosis of BOS which avoids the need for a histological diagnosis as the process can be patchy in the lung. This relies upon $\mathrm{FEV}_{1}$ and $F F_{25}-75$ measurements (table 6). Posttransplant, a baseline measurement is obtained based upon two readings taken 3 weeks apart without the use of inhaled bronchodilators. To diagnose BOS there must be a fall in $\mathrm{FEV}_{1}$ of $10-19 \%$ or a greater than $25 \%$ decrease in $\mathrm{FEF}_{25}-75$ and the drop must be sustained on two occasions more than 3 weeks apart [22].

High resolution CT scanning of the chest with inspiratory and expiratory views can prove useful in establishing the diagnosis and will typically show air trapping, bronchial wall thickening and central bronchiectasis.

Management of chronic rejection is by a multifactorial approach and may include a change in immunosuppression regime such as switching maintenance immunosuppressive drugs. Nonpharmacological immunosuppressive approaches have also been used including extracorporeal phototherapy (ECP) and total lymphoid irradiation (TLI). The exact mechanisms by which ECP or TLI may be beneficial in chronic rejection are not well described.

The intervention which has had the biggest impact in the management of chronic rejection is long-term use of the neomacrolide antibiotic azithromycin. It has been used as both a treatment for BOS and a potentially protective strategy. The first observational study into azithromycin as a treatment was performed in 2003 in six patients with BOS. Five of the six participants showed a significant improvement in $\mathrm{FEV}_{1}$ (approximately $500 \mathrm{~mL}$ ) compared with their baseline [23]. Further uncontrolled studies have upheld this as well as showing that it also has a positive effect on survival $[24,25]$. More recently a randomised controlled trial of azithromycin started 1 month after transplantation showed that its use was associated with a significant

Table 5. Classification of transbronchial lung biopsy results

\begin{tabular}{ll} 
Classification system & Interpretation \\
\hline Ao & No evidence of acute rejection \\
\hline$A_{1}$ & Minimal acute cellular rejection \\
\hline$A_{2}$ & Mild acute cellular rejection (treatment required) \\
\hline$A_{3}$ & Moderate acute cellular rejection \\
\hline$A_{4}$ & Severe acute cellular rejection \\
\hline Bo & No airway inflammation \\
\hline B1 $R$ & Mild airway inflammation \\
\hline B2R & Moderate-severe airway inflammation \\
\hline Co & No evidence of obliterative bronchiolitis \\
\hline
\end{tabular}

$\mathrm{C}_{1}$

Presence of obliterative bronchiolitis 
Table 6. Stages of bronchiolitis obliterans syndrome

\begin{tabular}{ll}
\hline BOS stage & Lung Function parameters \\
\hline BOS-O & $\mathrm{FEV}_{1}>90 \%$ and $\mathrm{FEF}_{25}-7575 \%$ of baseline \\
\hline BOS-op & $\mathrm{FEV}_{1} 81-90 \%$ and $\mathrm{FEF}_{25-75}<75 \%$ of baseline \\
\hline BOS-1 & $\mathrm{FEV}_{1} 66-80 \%$ of baseline \\
\hline BOS-2 & $\mathrm{FEV}_{1} 51-65 \%$ of baseline \\
\hline BOS-3 & $\mathrm{FEV}_{1}<50 \%$ of baseline
\end{tabular}

reduction in the incidence of chronic rejection compared with placebo [26].

\section{What new on the horizon for lung transplantation?}

The combination of increased availability of DCD lung donors and EVLP as a donor lung treatment platform offers substantial hope that the donor lung shortage might be resolved. This may open up lung transplantation to an increased number of patients with very advanced chronic lung disease.

The ability to support patients with acute (on chronic) respiratory failure without the need to intubate and invasively ventilate them by using newer extra-corporeal membrane oxygenation (ECMO) systems and with lung assist devices offers a tremendous opportunity to bridge potential lung transplant candidates to successful transplantation. This may also help reduce waiting list deaths by supporting patients until a donor organ is identified.

As the long-term respiratory function after lung transplantation continues to improve the challenges of managing the long-term complications of chronic immunosuppression usage increase. Cardiovascular and malignancy related morbidity and mortality now account for a major limitation to longterm survival. Immunosuppression regimes that lead to calcineurin inhibitor free management over time through induction of tolerance are needed in lung transplantation. Such approaches are currently being evaluated in other organ transplants.

\section{References}

1. Christie JD, Edwards LB, Kucheryavaya AY, et al. The Registry of the International Society for Heart and Lung Transplantation: 29th Adult Lung and HeartLung Transplant Report, 2012. J Heart Lung Transplant 2012; 31: 1073-1086.

2. Valapour M, Paulson K, Smith JM, et al. OPTN/SRTR 2011 Annual Data Report: Lung. Am J Transplant 2013; 13: 149-177.

3. Eurotransplant lung transplant statistics 2012. Available from: http://statistics.eurotransplant.org Date last updated: February 4th, 2013; date last accessed: February 18th, 2013

4. NHS Blood and Transplant UK Activity Report 20112012. Available from www.organdonation.nhs.uk/ statistics/transplant_activity_report/ Date last updated: March 31, 2012; date last accessed: February 18, 2012.

5. Orens JB, Estenne M, Arcasoy S, et al. International guidelines for the selection of lung transplant candidates: 2006 update - a consensus report from the ISHLT pulmonary scientific council. J Heart Lung Transplant 2006; 25: 745-755.
6. Celli BR, Cote CG, Marin JM, et al. The body mass index, airflow obstruction, dyspnea, and exercise capacity index in chronic obstructive pulmonary disease. New Engl J Med 2004; 350: 1005-1012.

7. Criner GJ, Cordova F, Sternberg AL, et al. The National Emphysema Trial (NETT) Part I; Lessons Learned about Emphysema. Am J Respir Crit Care Med 2011; 184: 763-770.

8. Chernenko SM, Humar A, Hutcheon M, et al. Mycobacterium abscessus infections in lung transplant recipients: the international experience. J Heart Lung Transplant 2006; 25: 1447-1455.

9. De Soyza A, Meachery G, Hester KL, et al. Lung transplantation for patients with cystic fibrosis and Burkholderia cepacia complex infection: a singlecenter experience. J Heart Lung Transplant 2010; 29: 1395-1404.

10. Mackay LS, Anderson RL, Parry G, et al. Pulmonary fibrosis: rate of disease progression as a trigger for referral for lung transplantation. Thorax 2007; 62: 1069-1073. 


\section{Suggested answers}

1. b, e

2. a, b, d, e

3. $a, b, e$

4. c, e
11. Orens JB, Boehler A, de Perrot $M$, et al. A review of lung transplant donor acceptability criteria - consensus document. J Heart Lung Transplant 2003; 22: 1183-1200.

12. Bonser RS, Taylor R, Collett D, et al. Effect of donor smoking on survival after lung transplantation: a cohort study of a prospective registry. Lancet 2012; 380: 747-755.

13. Steen S, Liao Q, Wierup PN, et al. Transplantation of lungs from non-heart-beating donors after functional assessment ex vivo. Ann Thorac Surg 2003; 76: 244-252.

14. Wierup P, Haraldsson A, Nilsson F, et al. Ex vivo evaluation of nonacceptable donor lungs. Ann Thorac Surg 2006; 81: 460-466.

15. Steen S, Ingemansson R, Eriksson L, et al. First human transplantation of a non acceptable donor lung after reconditioning ex vivo. Ann Thorac Surg 2007; 83: 2191-2194.

16. Singh N, Husain S. Aspergillus infections after lung transplantation: clinical differences in type of transplant and implication for management. J Heart Lung Transplant 2003; 22: 258-266.

17. Mooney ML, Hannan MM, Husain S, et al. eds. Diagnosis and Management of Infectious Diseases in Cardiothoracic Transplantation and Mechanical Circulatory Support. Maryland Heights, Elsevier, 2011.

18. Aris RM, Maia DM, Neuringer IP, et al. Posttransplantation lymphoproliferative disorder in the Epstein-Barr virus-naive lung transplant recipient. Am J Respir Crit Care Med 1996; 154: 1712-1717.
19. Snell GI, Boehler A, Glanville AR, et al. Eleven years on: a clinical update of key areas of the 1996 lung allograft rejection working formulation. J Heart Lung Transplant 2007; 26: 423-430.

20. Boehler A, Estenne M. Post-transplant bronchiolitis obliterans. Eur Respir J 2003; 22: 1007-1018.

21. Borthwick LA, Mcllroy El, Gorowiec MR, et al. Inflammation and epithelial to mesenchymal transition in lung transplant recipients: role in dysregulated epithelial wound repair. Am J Transplant 2010; 10: 498-509.

22. Estenne M, Maurer JR, Boehler A, et al. Bronchiolitis obliterans syndrome 2001: an update of the diagnostic criteria. J Heart Lung Transplant 2002; 21: 297-310.

23. Gerhardt SG, McDyer JF, Girgis RE, et al. Maintenance azithromycin therapy for bronchiolitis obliterans syndrome: results of a pilot study. $\mathrm{Am} J$ Respir Crit Care Med 2003; 168: 121-125.

24. Yates B, Murphy DM, Forrest IA, et al. Azithromycin reverses airflow obstruction in established bronchiolitis obliterans syndrome. Am J Respir Crit Care Med 2005; 172: 772-775.

25. Jain R, Hachem RR, Morrell MR, et al. Azithromycin is associated with increased survival in lung transplant recipients with bronchiolitis obliterans syndrome. J Heart Lung Transplant 2010; 29: 531-537.

26. Vos R, Vanaudenaerde BM, Verleden SE, et al. A randomised controlled trial of azithromycin to prevent chronic rejection after lung transplantation. Eur Respir J 2011; 37: 164-172. 